\title{
MAGIC Observations and multiwavelength properties of the quasar 3C 279 in 2007 and 2009
}

\begin{abstract}
J. Aleksić ${ }^{1}$, L. A. Antonelli ${ }^{2}$, P. Antoranz ${ }^{3}$, M. Backes $^{4}$, J. A. Barrio ${ }^{5}$, D. Bastieri ${ }^{6}$, J. Becerra González ${ }^{7,8}$, W. Bednarek ${ }^{9}$, A. Berdyugin ${ }^{10}$, K. Berger ${ }^{7,8}$, E. Bernardini ${ }^{11}$, A. Biland ${ }^{12}$, O. Blanch ${ }^{1}$, R. K. Bock ${ }^{13}$, A. Boller ${ }^{12}$, G. Bonnoli ${ }^{2}$, D. Borla Tridon ${ }^{13}$, I. Braun ${ }^{12}$, T. Bretz $^{14, \star}$, A. Cañellas ${ }^{15}$, E. Carmona $^{13}$, A. Carosi $^{2}$, P. Colin ${ }^{13}$, E. Colombo ${ }^{7}$, J. L. Contreras ${ }^{5}$, J. Cortina ${ }^{1}$, L. Cossio $^{16}$, S. Covino ${ }^{2}$, F. Dazzi ${ }^{16, \star \star}$, A. De Angelis ${ }^{16}$, E. De Cea del Pozo $^{17}$, B. De Lotto ${ }^{16}$, C. Delgado Mendez ${ }^{7, \star \star \star}$, A. Diago Ortega ${ }^{7,8}$, M. Doert $^{4}$, A. Domínguez ${ }^{18}$, D. Dominis Prester $^{19}$, D. Dorner ${ }^{12}$, M. Doro ${ }^{20}$, D. Elsaesser ${ }^{14}$, D. Ferenc ${ }^{19}$, M. V. Fonseca ${ }^{5}$, L. Font ${ }^{20}$, C. Fruck ${ }^{13}$, R. J. García López $^{7,8}$, M. Garczarczyk ${ }^{7}$, D. Garrido ${ }^{20}$, G. Giavitto ${ }^{1}$, N. Godinović ${ }^{19}$, D. Hadasch ${ }^{17}$, D. Häfner ${ }^{13}$, A. Herrero ${ }^{7,8}$, D. Hildebrand ${ }^{12}$, J. Hose ${ }^{13}$, D. Hrupec ${ }^{19}$, B. Huber ${ }^{12}$, T. Jogler ${ }^{13}$, S. Klepser ${ }^{1}$, T. Krähenbühl12 ${ }^{12}$ J. Krause ${ }^{13}$, A. La Barbera ${ }^{2}$, D. Lelas ${ }^{19}$, E. Leonardo ${ }^{3}$, E. Lindfors ${ }^{10}$, S. Lombardi ${ }^{6}$, M. López ${ }^{5}$, E. Lorenz ${ }^{12,13}$, P. Majumdar ${ }^{26}$, M. Makariev ${ }^{21}$, G. Maneva ${ }^{21}$, N. Mankuzhiyil ${ }^{16}$, K. Mannheim ${ }^{14}$, L. Maraschi ${ }^{2}$, M. Mariotti ${ }^{6}$, M. Martínez ${ }^{1}$, D. Mazin ${ }^{1,13}$, M. Meucci ${ }^{3}$, J. M. Miranda ${ }^{3}$, R. Mirzoyan ${ }^{13}$, H. Miyamoto ${ }^{13}$, J. Moldón ${ }^{15}$, A. Moralejo ${ }^{1}$, D. Nieto ${ }^{5}$, K. Nilsson ${ }^{10, \star \star \star \star}$, R. Orito ${ }^{13}$, I. Oya ${ }^{5}$, R. Paoletti ${ }^{3}$, S. Pardo ${ }^{5}$, J. M. Paredes ${ }^{15}$, S. Partini ${ }^{3}$, M. Pasanen ${ }^{10}$, F. Pauss ${ }^{12}$, M. A. Perez-Torres ${ }^{1}$, M. Persic ${ }^{16,22}$, L. Peruzzo ${ }^{6}$, M. Piliaa ${ }^{23}$, J. Pochon ${ }^{7}$, F. Prada ${ }^{18}$, P. G. Prada Moroni ${ }^{24}$, E. Prandini ${ }^{6}$, I. Puljak ${ }^{19}$, I. Reichardt ${ }^{1}$, R. Reinthal ${ }^{10}$, W. Rhode ${ }^{4}$, M. Ribó ${ }^{15}$, J. Rico ${ }^{25,1}$, S. Rügamer ${ }^{14}$, M. Rüger ${ }^{14}$, A. Saggion ${ }^{6}$, K. Saito ${ }^{13}$, T. Y. Saito ${ }^{13}$, M. Salvati ${ }^{2}$, K. Satalecka ${ }^{11}$, V. Scalzotto ${ }^{6}$, V. Scapin ${ }^{16}$, C. Schultz ${ }^{6}$, T. Schweizer ${ }^{13}$, M. Shayduk ${ }^{13}$, S. N. Shore ${ }^{24}$, A. Sillanpää ${ }^{10}$, J. Sitarek ${ }^{9}$, D. Sobczynska ${ }^{9}$, F. Spanier ${ }^{14}$, S. Spiro ${ }^{2}$, A. Stamerra ${ }^{3}$, B. Steinke ${ }^{13}$ J. Storz ${ }^{14}$, N. Strah ${ }^{4}$, T. Surić ${ }^{19}$, L. Takalo ${ }^{10}$, F. Tavecchio ${ }^{2}$, P. Temnikov ${ }^{21}$, T. Terzić19 ${ }^{19}$, D. Tescaro ${ }^{1}$, M. Teshima ${ }^{13}$, M. Thom ${ }^{4}$, O. Tibolla ${ }^{14}$, D. F. Torres ${ }^{25,17}$, A. Treves ${ }^{23}$, H. Vankov ${ }^{21}$, P. Vogler ${ }^{12}$, R. M. Wagner ${ }^{13}$, Q. Weitzel ${ }^{12}$, V. Zabalza ${ }^{15}$, F. Zandanel ${ }^{18}$, and R. Zanin ${ }^{1}$

(Affiliations can be found after the references)
\end{abstract}

Received 11 January 2011 / Accepted 18 February 2011

\section{ABSTRACT}

Context. 3C 279, the first quasar discovered to emit VHE $\gamma$-rays by the MAGIC telescope in 2006, was reobserved by MAGIC in January 2007 during a major optical flare and from December 2008 to April 2009 following an alert from the Fermi space telescope on an exceptionally high $\gamma$-ray state.

Aims. The January 2007 observations resulted in a detection on January 16 with significance $5.4 \sigma$, corresponding to a $F(>150 \mathrm{GeV})(3.8 \pm 0.8) \times$ $10^{-11} \mathrm{ph} \mathrm{cm}^{-2} \mathrm{~s}^{-1}$ while the overall data sample does not show significant signal. The December 2008-April 2009 observations did not detect the source. We study the multiwavelength behaviour of the source at the epochs of MAGIC observations, collecting quasi-simultaneous data at optical and X-ray frequencies and for 2009 also $\gamma$-ray data from Fermi.

Methods. We study the light curves and spectral energy distribution of the source. The spectral energy distributions of three observing epochs (including the February 2006, which has been previously published) are modelled with one-zone inverse Compton models and the emission on January 16, 2007 also with two zone model and with a lepto-hadronic model.

Results. We find that the VHE $\gamma$-ray emission detected in 2006 and 2007 challenges standard one-zone model, based on relativistic electrons in a jet scattering broad line region photons, while the other studied models fit the observed spectral energy distribution more satisfactorily.

Key words. quasars: individual: 3C 279 - gamma rays: galaxies

\section{Introduction}

3C 279 was the first quasar discovered as a $\gamma$-ray source with the Compton Gamma-Ray Observatory (Hartman et al. 1992), and the first flat-spectrum radio quasar (FSRQ) discovered to emit

\footnotetext{
^ Now at: École Polytechnique Fédérale de Lausanne (EPFL), Lausanne, Switzerland.

$\star \star$ Supported by INFN Padova.

$\star \star \star$ Now at: Centro de Investigaciones Energéticas, Medioambientales y Tecnológicas (CIEMAT), Madrid, Spain.

$\star \star \star \star$ Now at: Finnish Centre for Astronomy with ESO (FINCA), Turku, Finland.
}

very high energy (VHE, defined here $>100 \mathrm{GeV}$ ) $\gamma$-rays (Albert et al. 2008a). With a redshift of 0.536 (Hewitt \& Burbridge 1993), 3C 279 is also the most distant of the VHE $\gamma$-ray emitting sources discovered so far.

$3 \mathrm{C} 279$ is one of the brightest quasars at all wavelengths and its multiwavelength behavior and jet structure has been studied in great detail (e.g. Hartman et al. 2001; Chatterjee et al. 2008). Its relativistic jet, which is the source of the radio to VHE $\gamma$-ray emission, is closely aligned with the line of sight (the angle varies, but is sometimes as small as $<0.5^{\circ}$, Jorstad et al. 2004). The radio to optical emission is synchrotron radiation emitted by the relativistic electrons spiraling in the magnetic field of 
the jet. In this low-energy regime the total flux density variations are well described by shocks propagating in the jet (e.g. Lindfors et al. 2006). The X-ray emission can be explained by the synchrotron self-Compton mechanism (SSC, e.g. Maraschi et al. 1992; Hartman et al. 2001; Sikora et al. 2001), where the synchrotron photons emitted by the jet act as seed photons for the inverse Compton scattering.

However, there is no consensus about the emission mechanism and site of the $\gamma$-ray and VHE $\gamma$-ray emission in 3C 279. The emission can, in principle, be explained by both leptonic and hadronic models: the leptonic models mostly rely on external Compton (EC, e.g. Hartman et al. 2001; Albert et al. 2008a), invoking the inverse Compton scattering of external photons from accretion disk or broad line region (BLR) clouds (Dermer \& Schlickeiser 1993; Sikora et al. 1994), while in the hadronic models (Mannheim \& Biermann 1992; Mücke et al. 2003; Böttcher et al. 2009) the VHE $\gamma$-ray photons are produced by proton initiated cascades or directly through proton synchrotron radiation (but see Sikora et al. 2009, for criticisms).

The leptonic models are very sensitive to the site of the emission: the external Compton models relying on photons originating from broad line emission clouds are not efficient if the emitting blob is outside the BLR and in the SSC models the $\gamma$-ray emission must originate from a different emission region than the main component of the synchrotron radiation in order to reproduce the observed $\gamma$-ray flux (Böttcher et al. 2009). It should also be noted, that independently of the emission mechanism the internal absorption cannot be neglected if the emission region is located inside the BLR (Sitarek \& Bednarek 2008; Tavecchio $\&$ Mazin 2009). Alternatively, the emission can be produced in regions located far beyond the broad line region, at distances at which the dominant radiation field for EC is that of the parsecscale dusty torus (Sikora et al. 2008). In this case, internal absorption can be neglected up to $\sim 1 \mathrm{TeV}$ but, due to the large size of the emission region, a minimum variability timescale of the order of $\sim 1$ day is expected.

Amongst Fermi detected blazars (Abdo et al. 2009) hard overall spectrum (spectral index $=2.32$ ) but also the comparatively weak evidence of a break in the spectrum at $1 \mathrm{GeV}$. The spectral index after the break energy is given as 2.50 without apparent cut-off. This is one of the hardest spectrum of all bright FSRQs above a few GeV and makes it one of the prime target for VHE $\gamma$-ray observations.

In this paper we present VHE $\gamma$-ray observations of 3C 279 performed by the MAGIC-I telescope in January 2007 and from December 2008 to April 2009. The 2007 observations were triggered by an optical outburst in the source while the December 2008 observations were triggered by an alert from the Fermi space telescope. The January-April 2009 data were taken as a part of the followed multiwavelength campaign. We present the data analysis and results of the MAGIC observations, together with simultaneous multiwavelength observations and a discussion of theoretical models. For comparison and completeness we also summarize the 2006 observation campaign (Albert et al. 2008a). The plan of the paper is as follows. The MAGIC observations, data analysis and results are presented in Sect. 2. Multiwavelength observations are presented in Sect. 3 and discussed in Sect. 4 together with the MAGIC data. VHE $\gamma$-ray and multifrequency data are combined in Sect. 5 to build quasisimultaneous spectral energy distributions at different epochs and theoretical models are presented and critically discussed. A summary of results and conclusions are given in Sect. 6 .

\section{MAGIC observations}

The data described in this paper were taken with the first MAGIC (Major Atmospheric Gamma-ray Imaging Cherenkov) telescope as a standalone imaging atmospheric Cherenkov telescope. It is located on the Canary Island of La Palma. MAGIC has a standard trigger threshold of $60 \mathrm{GeV}$ for observations at low zenith angles, an angular resolution of $\sim 0.1^{\circ}$ on the event by event basis and an energy resolution above $150 \mathrm{GeV}$ of $\sim 25 \%$ (see Albert et al. 2008b, for details).

MAGIC observed 3C 279 in January 2007 and from December 2008 to April 2009. Due to changes in the telescope systems these data sets were analyzed separately.

\subsection{January 2007}

3C 279 was observed during nine nights in January 2007 for a total of $23.6 \mathrm{~h}, 18.6 \mathrm{~h}$ (seven nights) of which passed the quality selection. The observations were conducted in moon, dark night and twilight. The zenith angle range of the observations was 35 to 46 degrees. The readout chain included a fast $300 \mathrm{MSample} / \mathrm{s}$ FADC system, which allows us to use the time information of the showers in the analysis as described in Aliu et al. (2009). Since the average night sky background noise level stayed below 3 times the dark time level, all data has been analyzed with the same standard image cleaning levels as discussed in Britzger et al. (2009). The data were taken in On mode where the telescope is pointing directly towards the source. So-called Off data were taken under similar conditions (zenith angle, trigger rates and weather conditions) from September 2006 until January 2007 and has been used to estimate the background level. Due to the installation of the new $2 \mathrm{GHz}$ MUX-FADC read-out system in 2007 February, no Off data after January 2007 were fulfilling the requirement of similar observation and hardware conditions.

The 2007 data were analyzed using so-called Würzburg Analysis chain described in detail in Bretz \& Wagner (2003) and Bretz \& Dorner (2008). The data were calibrated following the description in Albert et al. (2008c), the signal was extracted at the pulse maximum using a spline method, the air-shower images were cleaned of noise from night-sky background light by applying a time image cleaning. In the first step a minimum number of 6 photo-electrons in two adjacent pixels is required (so called "core pixels"). Each pixel next to a core pixel, which is above a threshold of 3 photo-electrons is considered a "boundary pixel". Additionally to these limits in the charge of the pixels it is required that the arrival time of core pixels is within $1.75 \mathrm{~ns}$ of the mean arrival time of all core pixels and that boundary pixels arrive within $4.5 \mathrm{~ns}$ of the mean arrival time of the core pixels. Both the charge and the time limits have to be fulfilled, otherwise the pixel is considered to contain noise and deleted from the image. We parametrized the shower images (Hillas 1985) and used a SIZE-dependent parabolic cut in AREA, WIDTH and LENGTH (Riegel et al. 2005) for the $\gamma$ /hadron separation. The cut parameters were optimized on Crab Nebula data taken within the same zenith angle range as the 3C 279 data. The energy threshold of the analysis was $220 \mathrm{GeV}$. The arrival direction of the $\gamma$-rays is reconstructed using the DISP method (Fomin et al. 1994; Lessard et al. 2001), which was adapted to use the shower timing information as described in Aleksić et al. (2010). The significance of a detection is evaluated by comparison of the number of events in the On and the Off data sample using formula 17 of $\mathrm{Li} \& \mathrm{Ma}(1983)$. 
Table 1. Night-by-night results of MAGIC January 2007 observations of 3C 279.

\begin{tabular}{lllll}
\hline \hline Observation night [MJD] & Observation time [min] & Excess events [counts] & Background events [counts] $^{*}$ On/Off scaling factor ${ }^{1}$ & Significance $^{2}$ \\
\hline 54115 & 149.5 & 17.6 & 97.4 & 0.058 \\
54116 & 151.1 & 64.1 & 102.9 & 0.062 \\
54117 & 157.2 & -7.9 & 100.9 & 0.060 \\
54118 & 153.6 & -6.0 & 82.0 & 0.049 \\
54120 & 164.1 & -12.3 & 117.3 & 0.07 \\
54121 & 166.7 & 12.7 & 119.3 & 0.07 \\
54122 & 175.1 & 18.2 & 126.8 & -0.8 \\
\hline
\end{tabular}

Notes. ${ }^{(1)}$ The scaling factor is the ratio between the on and the off $\theta$ distribution normalized outside the signal region. ${ }^{(2)}$ Significance is given as standard deviations and is not corrected for seven trials, arising from diving the data sample by seven (corresponding to seven nights with good quality data).

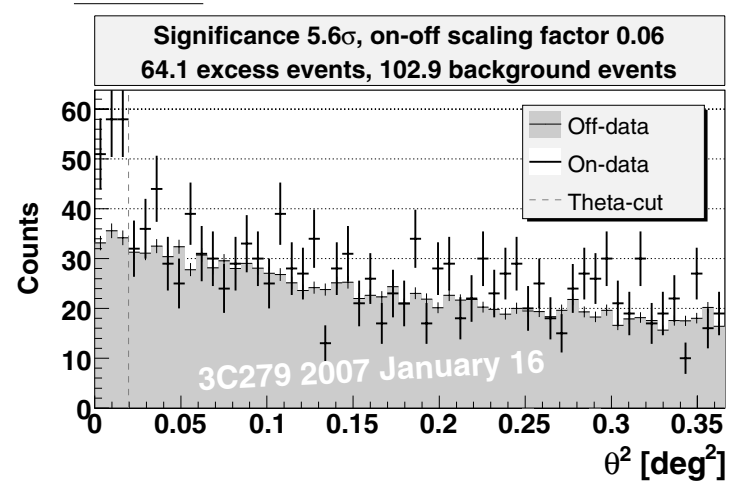

Fig. 1. Reconstructed shower direction $\theta^{2}$ for the Off (gray shaded area) and On (black crosses) data as observed on January 16, 2007 by MAGIC. The vertical dotted line corresponds to the apriori defined signal region. An excess of 64 events is clearly visible in the On data as compared to the normalized Off data. The corresponding pre-trial significance is $5.6 \sigma$ and $5.4 \sigma$ after correction for seven trials, respectively.

Since in 2006 the source was detected in a single day flare, every observation day was analyzed separately. Table 1 summarizes the analysis results of the January 2007 campaign. Out of the seven observation nights with good data quality only one, MJD 54116 (January 16), revealed a significant excess in the MAGIC data after Off-subtraction. The significance of the excess is $5.6 \sigma$ (pre-trial) with 64.1 excess events above 102.9 normalized Off events in $151 \mathrm{~min}$ of observations. The $\theta^{2}$ (squared distance between the true and reconstructed source position, see e.g. Daum et al. 1997) distribution is shown in Fig. 1. None of the other observation nights yielded in a significant excess.

For spectrum (Fig. 2) and light curve softer cuts that have a higher $\gamma$-ray efficiency were used. The VHE $\gamma$-ray spectrum of the flare can be described by a simple power law (with the differential flux given in units of $\mathrm{TeV}^{-1} \mathrm{~m}^{-2} \mathrm{~s}^{-1}$ ):

$\frac{\mathrm{d} F}{\mathrm{~d} E}=(5.7 \pm 1.3) \times 10^{-7}\left(\frac{\mathrm{E}}{300 \mathrm{GeV}}\right)^{-3.1 \pm 1.1}$.

The highest spectral energy point has a medium energy of $350 \mathrm{GeV}$. For a Crab like spectrum we have a systematic energy scale error of $16 \%$, a systematic error of $11 \%$ on the flux normalization (without the energy scale error), and a systematic slope error of 0.2 (a detailed list of all the contributions can be found in Albert et al. 2008b), while for the soft spectrum like 3C 279 the systematic errors are expected to be slightly larger. Assuming that 3C 279 always emits $\gamma$-rays above $150 \mathrm{GeV}$ we calculated the corresponding light curve (see Sect. 4) on the night by night basis. For January 16th the integral flux above $150 \mathrm{GeV}$ is $(3.8 \pm 0.8) \times 10^{-10} \mathrm{ph} \mathrm{cm}^{-2} \mathrm{~s}^{-1}$.

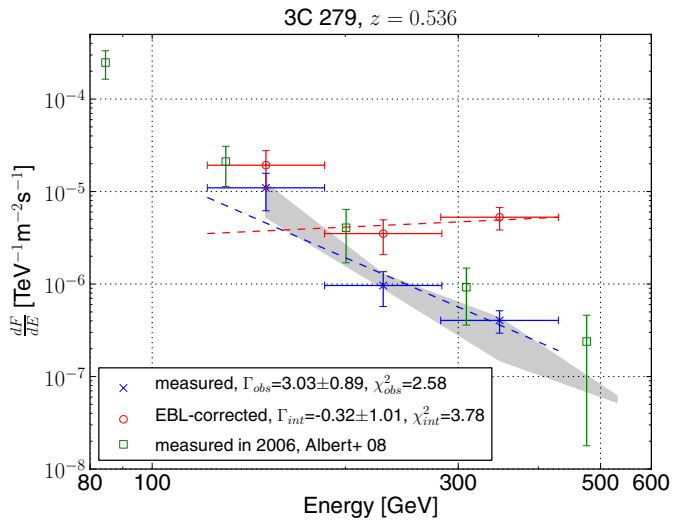

Fig. 2. Spectrum of the VHE $\gamma$-ray flare January 16, 2007. The blue points correspond to the measured spectrum with the respective errors on the flux and the bin size in energy. The results of a simple power law fit are shown as blue line. The gray shaded region represents the systematic error of the analysis. The red points show the spectrum deabsorbed using the EBL model by Dominguez et al. (2010) and the red dashed line the fit to this EBL corrected spectrum. For comparison the spectrum from 2006 (Albert et al. 2008a) is shown with green points.

The results were cross-checked using the standard MAGIC analysis chain (Albert et al. 2008c). The standard analysis resulted in lower significance, $4.6 \sigma$, for the detection. For flux and spectral index the results were comparable for both analysis chains.

\subsection{December 2008-April 2009}

MAGIC observations started on December 9, 2008 after the Fermi Collaboration announced a high $\mathrm{GeV} \gamma$-ray state of the source (Ciprini et al. 2008). Unfortunately 3C 279 could only be observed at zenith angles larger than 46 degrees and for a short time $(\sim 10 \mathrm{~min})$ due to visibility constraints. These short runs were insufficient to detect the source. Follow-up observations were conducted at the end of December 2008 until April 2009 under more favorable zenith angles, smaller than 35 degrees.

A total observation time of $28.1 \mathrm{~h}$ was accumulated over 20 days with the $2 \mathrm{GHz}$ MUX-FADC read-out system. $35 \mathrm{~min}$ were recorded in On-mode, while the remaining data $(27.5 \mathrm{~h})$ were taken in wobble mode where the source was displaced by $0.4^{\circ}$ from the camera center in order to allow the recording of simultaneous Off data with the same offset from the camera center (Daum et al. 1997). After quality selection $11.9 \mathrm{~h}$ of data were used in the analysis. The main part of this data 
Table 2. Night-by-night results of MAGIC December 2008-April 2009 observations.

\begin{tabular}{llllll}
\hline \hline Observation night [MJD] & Observation time [min] & Excess events [counts] & Background events [counts] & On/Off scaling factor $^{1}$ & Significance $^{2}$ \\
\hline 54829 & 50.4 & -0.3 & 65.3 & 0.03 & -0.04 \\
54832 & 60.0 & -10.7 & 92.7 & 0.02 & -0.98 \\
54834 & 81.0 & 4.8 & 103.2 & 0.02 & 0.41 \\
54838 & -1.4 & 48.4 & 0.08 & -0.18 \\
54852 & 78.0 & 12.9 & 42.1 & 0.07 & 1.73 \\
54855 & 33.6 & 4.1 & 241.9 & 0.06 & 0.24 \\
54856 & 5.3 & 70.7 & 0.10 & 0.57 \\
54937 & 157.8 & -0.3 & 110.3 & 0.03 & -0.03 \\
\hline
\end{tabular}

Notes. ${ }^{(1)}$ The scaling factor is the ratio between the on and the off $\theta$ distribution normalized outside the signal region. ${ }^{(2)}$ Significance is given as standard deviations and is not corrected for trials, arising from diving the data sample by eight (corresponding to eight nights with good quality data).

Table 3. Differential upper limits (95\% confidence level) for December 2008-April 2009 observations.

\begin{tabular}{ll}
\hline \hline Energy bin $[\mathrm{GeV}]$ & Upper limit $\left[1 / \mathrm{TeV} / \mathrm{m}^{2} / \mathrm{s}\right]$ \\
\hline $150-200$ & $1.8 \times 10^{-5}$ \\
$200-315$ & $1.6 \times 10^{-6}$ \\
$315-430$ & $7.2 \times 10^{-7}$ \\
$430-580$ & $2.5 \times 10^{-7}$ \\
\hline
\end{tabular}

set is from January 2009, but it also includes one night (29) in December 2008 and one (16) in April 2009.

The data were analyzed using the standard analysis chain as described in Albert et al. (2008b,c) and Aliu et al. (2009). In order to suppress the background showers produced by charged cosmic rays, a multivariate classification method known as Random Forest is used (Albert et al. 2008d). For every event, the algorithm takes as input a set of image parameters, and produces one single parameter as output, called HaDronNEss. The background rejection is then achieved by a cut in HadronNess, which was optimized using Crab Nebula data taken under comparable conditions.

A cut in $\theta^{2}$, was used to extract the signal and also optimized in the same way. An additional cut removed the events with a total charge of less than 200 photo-electrons (phe) in order to assure a better background rejection.

We find no signal in this data set. The number of excess events is 29 over a background of 775 events, corresponding to a significance of $0.94 \sigma$. The night by night results are reported in Table 2.

All data were combined for the calculation of flux upper limits in the energy range between $150 \mathrm{GeV}$ and $580 \mathrm{GeV}$ (higher energies can be neglected due to the strong EBL absorption). Including a $30 \%$ systematic error and assuming a power law with a spectral index of -4 the integrated flux upper limit $(2 \sigma$ c.l.) is $6.3 \times 10^{-7} \mathrm{ph} / \mathrm{m}^{2} / \mathrm{TeV} / \mathrm{s}$. In Table 3 the differential values are reported.

\section{Multiwavelength observations}

We present here long term optical monitoring data from the Tuorla blazar monitoring program, and near infrared data from REM (for January 2007), X-ray data from SWIFT (for February 1, 2009) and $\gamma$-ray data from Fermi (for January 2009).

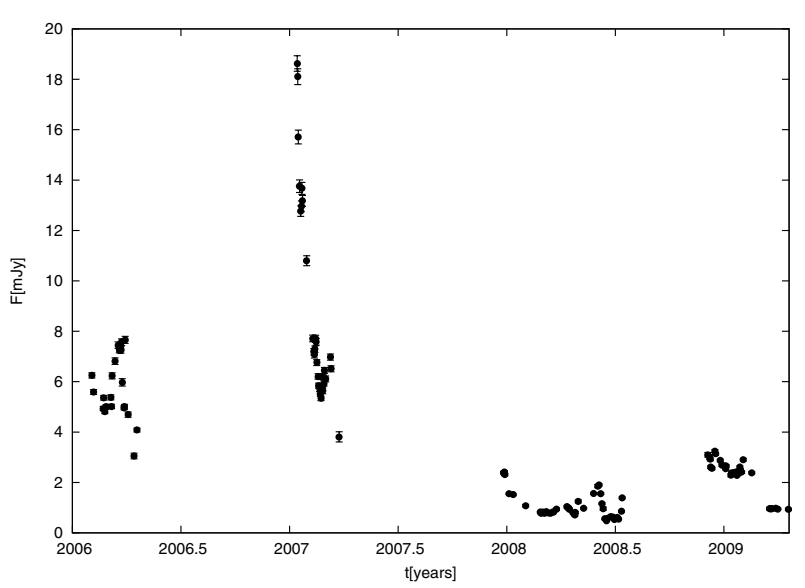

Fig. 3. Long term optical $R$-band observations of the Tuorla blazar monitoring program of 3C 279. The giant flare near the beginning of 2007 is clearly visible.

\subsection{Optical observations}

3C 279 has been observed regularly since 2004 as a part of the Tuorla blazar monitoring program ${ }^{1}$. Figure 3 shows the long term optical $R$-band light curve covering all the MAGIC observation periods discussed in this paper. In 2009 also polarimetric observations of 3C 279 were performed. The photometric optical $R$-band observations are performed with the Tuorla $1 \mathrm{~m}$ telescope (Finland) and Kungliga Vetenskapsakademien (KVA) $35 \mathrm{~cm}$ telescope (La Palma). The latter can be controlled remotely from Tuorla Observatory.

KVA consists of two telescopes, the larger one being a $60 \mathrm{~cm}$ $(f / 15)$ Cassegrain telescope equipped with a CCD polarimeter capable of polarimetric measurements in BVRI-bands using a plane-parallel calcite plate and a super-achromatic lambda/2 retarder. For 3C 279 the observations were done without filter to improve the signal-to-noise of the observations.

The $35 \mathrm{~cm}$ telescope is used for photometric measurements in $B, V$ and $R$-bands. The photometric measurements are made in differential mode, i.e. by obtaining CCD images of the target and calibrated comparison stars in the same field of view (Raiteri et al. 1998). The magnitudes of the source and comparison stars are measured using aperture photometry and the (color corrected) zero point of the image determined from the comparison star magnitudes. Finally, the object magnitude is computed using the zero point and a filter-dependent color correction. Magnitudes are then transferred to linear fluxes using the

${ }^{1}$ http://users.utu.fi/kani/1m/ 
formula $F=F_{0} \times 10^{\mathrm{mag} /-2.5}$, where mag is the object magnitude and $F_{0}$ is a filter-dependent zero point (in the $R$-band the value $F_{0}=3080 \mathrm{Jy}$ is used from Bessell et al. 1979).

For polarimetric measurements the normalized Stokes parameters and the degree of polarization and position angle were calculated from the intensity ratios of the ordinary and extraordinary beams using standard formula (e.g. Degl'Innocenti et al. 2007) and semiautomatic software specially developed for polarization monitoring purposes. During some of the nights, polarized standard stars from Turnshek et al. (1990) were observed to determine the zero point of the position angle. The instrumental polarization of the telescope has been found to be negligible.

\subsection{REM observations in January 2007}

In January 2007 3C 279 was intensively monitored by REM in the $I R$ band ( $J, H$ and $K$ filters). REM is a $60-\mathrm{cm}$ diameter fast-reacting telescope with $10^{\circ} \mathrm{s}^{-1}$ pointing speed located at the Cerro La Silla premises of the European Southern Observatory, Chile (Zerbi et al. 2001; Chincarini et al. 2003; Covino et al. 2004b). The telescope hosts REMIR, an infrared imaging camera, and ROSS, an optical imager and slitless spectrograph. The two cameras observe simultaneously the same field of view of $10^{\prime} \times 10^{\prime}$ thanks to a dichroic mirror, although only the infrared data are reported here. The telescope was designed to rapidly follow transient high-energy events as Gamma-Ray Bursts (GRBs) but in the idle time it is perfectly suited for monitoring programs of variable sources. The observations, including the short and long-term scheduling, are totally automatic. Typical exposure durations were of $150 \mathrm{~s}$ in the $J, H$ and $K$ s filters. Data were reduced in a standard way by means of tools provided by the ESO-Eclipse package (Devillard 1997). Standard aperture photometry was derived and results calibrated by a suitable number of well-exposed 2MASS objects in the field ${ }^{2}$.

\subsection{Swift and Fermi observations in January-February 2009}

The Swift satellite (Gehrels et al. 2004) is a NASA mission, launched in 2003. Swift performed Target of Opportunity observation of 3C 279 on February 1, 2009.

Swift/XRT is a Wolter type I grazing incidence telescope, with $110 \mathrm{~cm}^{2}$ effective area, 23.6' arcmin FOV and 15" angular resolution, sensitive in the $0.2-10 \mathrm{keV}$ energy band. Data were reduced using the software distributed with the heasoft 6.3.2 package by the NASA High Energy Astrophysics Archive Research Center (HEASARC). The xrtpipeline was set for the photon counting or window timing modes and having selected single pixel events (grade 0). Data shown in the SEDs were rebinned in order to have at least 30 counts per energy bin. Power law models have been fitted to the spectra. The X-ray reddening due to absorbing systems along the light travel path has been corrected assuming the Galactic value for column density of neutral hydrogen $N_{\mathrm{H}}=2.1 \times 10^{20} \mathrm{~cm}^{-2}$ (Kalberla et al. 2005).

UVOT is a $30 \mathrm{~cm}$ diffraction limited optical-UV telescope, equipped with 6 different filters, sensitive in the 1700-6500 wavelenght range, in a $17^{\prime} \times 17^{\prime}$ FOV. During the pointing of February 1, 2009 used below for the SED, only filters $U V W 1$ and $U$ were available. Analysis was performed by means of the uvotimsum and uvotsource tasks with a source region of $5^{\prime \prime}$, while the background was extracted from a source-free circular region with radius equal to $50^{\prime \prime}$ (it was not possible to use an annular region, because of a nearby source). The extracted $v F_{v}$

\footnotetext{
${ }^{2}$ http://www.ipac.caltech.edu/2mass/
}

magnitudes have been corrected for Galactic extinction using the values of Schiegel et al. (1998) and applying the formulae by Pei (1992) for the $U V$ filters, and finally converted into fluxes following Poole et al. (2008).

The Fermi LAT is a pair conversion telescope designed to cover the energy band from $20 \mathrm{MeV}$ to greater than $300 \mathrm{GeV}$ which operates in all-sky scanning mode.

The average LAT spectrum was derived in the period of the MAGIC pointings using the publicly available LAT data ${ }^{3}$. The photons of class 3 (DIFFUSE) with energy in the range 0.1-100 GeV collected from January 21, 2009 to January 31, 2009 were selected. These data were processed by using Science Tools 9.15.2, which includes the Galactic diffuse and isotropic background and the Instrument Response Function IRF P6_V3_DIFFUSE. Then photons in the good-time intervals and within a region of interest (ROI) with radius of $10^{\circ}$ from the source radio position were selected and a cut on the zenith angle parameter $\left(<105^{\circ}\right)$ to avoid the Earth albedo was applied. The following steps were to calculate the live-time cube, the exposure map and the diffuse response.

With all these information at hands, an analysis by using an unbinned likelihood algorithm (gtlike) in separate energy bins was performed. The model included the isotropic and Galactic diffuse backgrounds, the source of interest, all the 1FGL sources in the ROI and, possibly, additional sources not included there but identified in the map. For all the point sources we assumed a power law spectrum, with flux and photon index as a free parameter and calculated the corresponding test statistic ( $T S$, see Mattox et al. 1996, for a definition; in practice one assumes $\sqrt{T S} \simeq \sigma$, the significance of the detection). In the SED we report the four bins with high significance, $T S>25$.

\section{Multiwavelength behavior}

In addition to multiwavelength data discussed in the previous section, there are also previously published multiwavelength data for all the MAGIC observing epochs (spring 2006; Böttcher et al. 2007; Collmar et al. 2010; winter 2006-2007; Larionov et al. 2008, 1996-2007; Chatterjee et al. 2008, winter 2008spring 2009; Abdo et al. 2009). In the following we discuss the multiwavelength behaviour of the source in these three epochs comparing the data described in this paper to previously published results.

In the spring of 2006 when 3C 279 was first discovered by MAGIC, the source was in a high optical and X-ray state. However no optical or X-ray flare simultaneous to the VHE $\gamma$-ray flare was observed (Fig. 4). We are not aware of any optical polarization monitoring data for this epoch. In the radio bands the source was in quiescent state (Böttcher et al. 2007; Collmar et al. 2010) and there was no coincident very long baseline interferometry (VLBI) knot emerging from the VLBI core (Chatterjee et al. 2008), suggesting that the VHE $\gamma$-ray emission originates from region that is opaque to radio frequencies.

In January 2007 3C 279 was very bright in the optical band, reaching a peak flux of $19 \mathrm{mJy}(R \sim 13)$ on MJD 54114 . This is the maximum flux observed from the source in five years of Tuorla blazar monitoring. However, during the MAGIC observation the optical flux was already decreasing and on the night of January 16, 2007 (MJD 54 116) no increase in the optical flux was detected. A similar behavior was seen in the nearIR band, with a clear peak at January 13 followed by a decay. Unfortunately REM did not observe 3C 279 on January 15

\footnotetext{
3 Accessible from http://fermi.gsfc.nasa.gov
} 

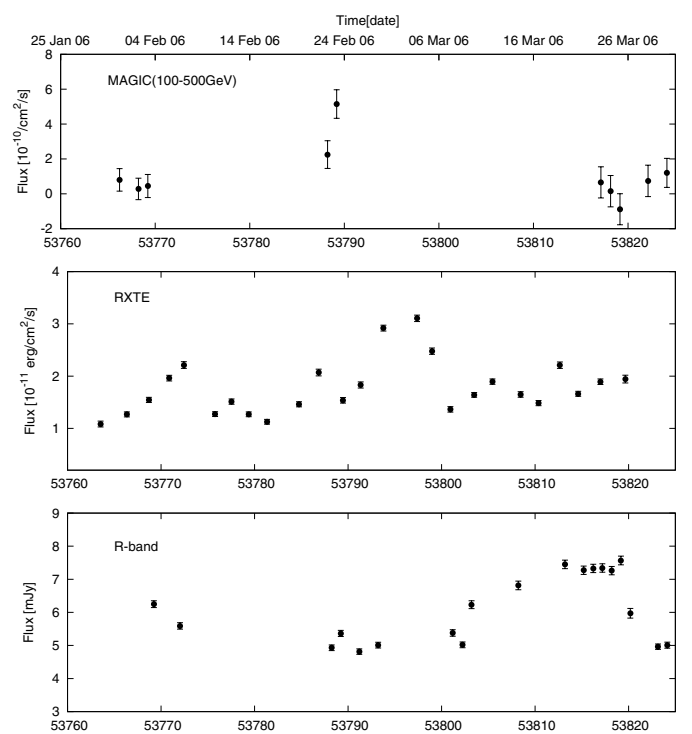

Fig. 4. Light curve in different energy bands during the 2006 observation campaign. From top to bottom: VHE $\gamma$-ray flux between $100-500 \mathrm{GeV}$ as measured by MAGIC (Albert et al. 2008a), RXTE PCA flux in the $2-10 \mathrm{keV}$ range (Chatterjee et al. 2008) and KVA $R$-band observations.

and 16 , but both on January 14 and 17 the data show a steep spectrum, smoothly joining with the $R$-band measure of KVA. Therefore, it is reasonable to assume that also the IR flux decreased smoothly during the VHE $\gamma$-ray flare.

The fluxes inferred from the MAGIC $(>150 \mathrm{GeV})$, the RXTE PCA (2-10 keV), the KVA ( $R$-band) and REM near infrared observations of January 2007 are compared as a function of time (light curves) in Fig. 5. As can be seen in Fig. 5, the VHE $\gamma$-ray flare does not coincide with neither the maximum of the optical flux or the maximum of the X-ray flux that were both observed prior to MAGIC observations. A comparison of the different light curves suggests a possible lag of 2-3 days of the VHE $\gamma$-ray flare with respect to the lower frequencies. It should also be noted, that the X-ray flux is in general lower than during the MAGIC observations in 2006.

In 2006-2007 there was also extensive whole earth blazar telescope (WEBT), very long baseline array (VLBA) and RXTE X-ray satellite monitoring of 3C 279 (Larionov et al. 2008). The VLBA data shows a component emerging from the core at MJD $54063 \pm 40$ accompanied by the maxima in the $37 \mathrm{GHz}$ light curve while the optical polarization data shows a rotation of the polarization angle $\sim 300^{\circ}$ starting around MJD 54115 with duration of $\sim 2$ months. There is coincident rotation of the polarization angle of the VLBI $43 \mathrm{GHz}$ core and thus Larionov et al. (2008) conclude that the optical flares and rotation of the optical polarization angle take place in the VLBI knot as it moves downstream of the core. The timing of the MAGIC detection (MJD 54 116) suggests that the emission of the VHE $\gamma$-rays also happens in this same emission region. This would be in agreement with previous results, that the $\gamma$-ray flares observed by EGRET and Fermi would take place in the knots freshly emerging from the VLBI core (see Jorstad et al. 2001; Jorstad et al. 2010; Agudo et al. 2011). This is sometimes denoted as "far dissipation" scenario, as the VLBI core is located tens of parsecs from the central engine (e.g. Marscher et al. 2010). However, it has been argued (e.g. Tavecchio et al. 2010) that the size of the emission region at this distance from the central engine is rather large which might be in contradiction with
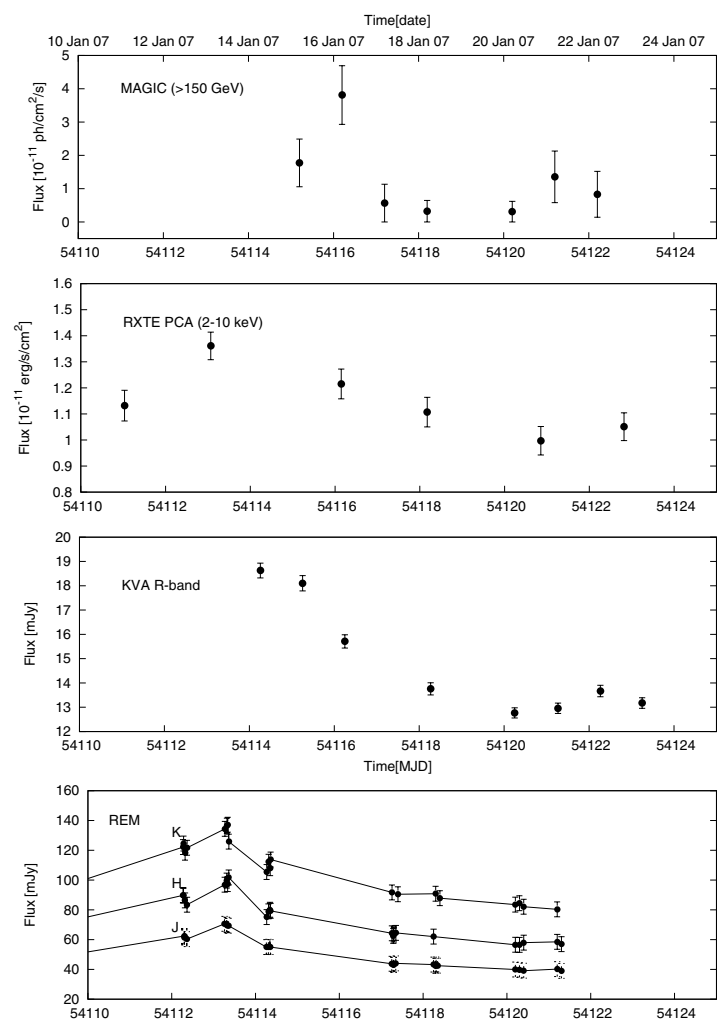

Fig. 5. Light curve in different energy bands during the 2007 observation campaign. From top to bottom: VHE $\gamma$-ray flux above $150 \mathrm{GeV}$ as measured by MAGIC, RXTE PCA flux in the $2-10 \mathrm{keV}$ range (Chatterjee et al. 2008), KVA R-band observations and REM infrared observations. For the MAGIC light curve the night by night flux is calculated assuming that 3C 279 always emits $\gamma$-rays above $150 \mathrm{GeV}$.

the day scale variability of the MAGIC observation. This is further discussed in the following sections.

In December 2008-January 2009 the source was in a quiescent state in the optical band. The polarization degree remained rather high $(\sim 30 \%)$ during the MAGIC observations and the polarization angle was rotating smoothly from 60 to 90 degrees. The light curves are shown in Fig. 6. In the X-ray band the source was at quiescent level, the flux being about $50 \%$ lower than in January 2007 (Abdo et al. 2009). In the $\gamma$-ray band (Fermi) the source was active from mid-November 2008 to the end of February 2009 and showed two major flares (first one peaking end of November (MJD 54795) and second mid February (MJD 54880 )), but the core of the MAGIC observations in 2009 January (MJD 54 829-54 856) took place in between the two flares, when the $\gamma$-ray flux was at a relatively low level. Unfortunately, due to bad weather on the MAGIC site, no data could be taken in February, when Fermi observed the $\gamma$-ray flare which was accompanied by a rotation of the optical polarization angle nor at the end of April when the source was flaring in the X-ray band (Abdo et al. 2009).

The optical to VHE $\gamma$-ray data of the three MAGIC observing epochs is summarized in Fig. 7. In summary, although the detections at VHE $\gamma$-ray energies correspond to high states in optical and X-rays, no multiwavelength correlations on short timescales seem to be present. 

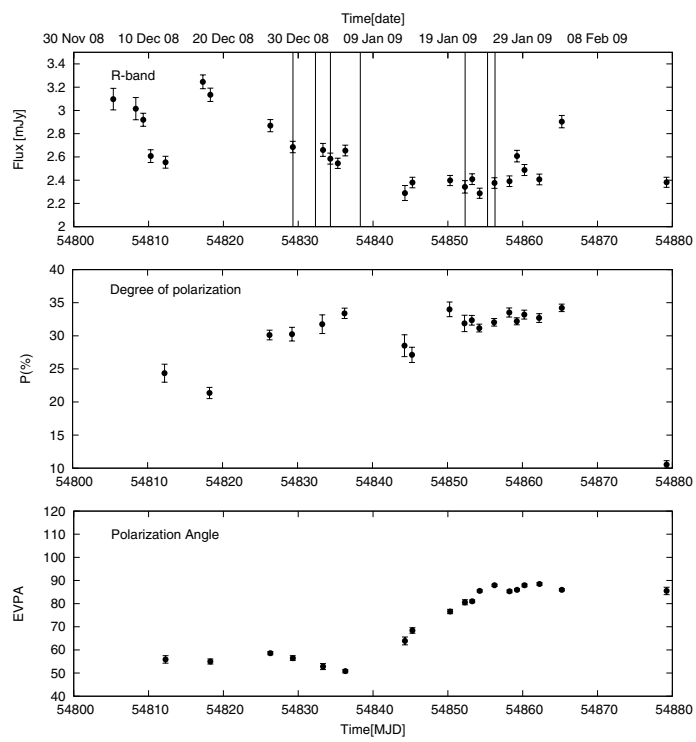

Fig. 6. The optical $R$-band light curve (top), degree of polarization (middle) and polarization angle of 3C 279 from 2008 December to 2009 February. The vertical lines show the time of MAGIC observations.

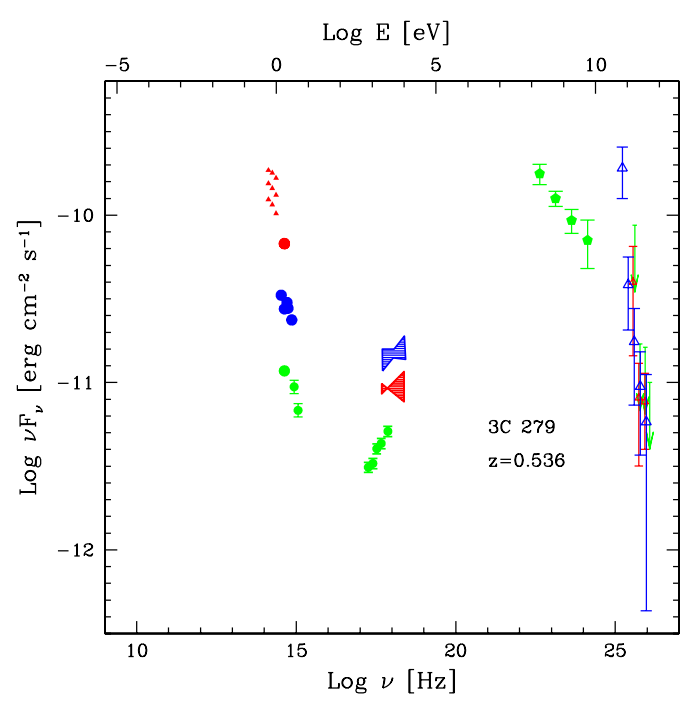

Fig. 7. SEDs of simultaneous optical, X-ray and $\gamma$-ray data at the epochs of MAGIC observations. Blue: February 23, 2006, red: January 16, 2007, green January 21-February 12009.

\section{Spectral energy distributions and models}

In the following we discuss the spectral energy distributions (SEDs) of 3C 279 at three epochs, February 23, 2006, January 16, 2007 and January 21-February 1, 2009. The first epoch corresponds to the MAGIC discovery of the VHE $\gamma$-ray emission from 3C 279 reported in Albert et al. (2008a). In addition to the multiwavelength data discussed there we show IR, optical and X-ray data simultaneous to the MAGIC observations from Böttcher et al. (2009). For January 16, 2007 and January 21-31, 2009 we show the quasi-simultaneous data described in Sect. 3.

The optical-IR data are important for SED modeling, since the steep continuum revealed by these measurements constrain the position of the synchrotron peak below the optical band in 2006 and below the IR band in 2007. The X-ray spectral data are also quite constraining, since they allow us to define the low energy end of the inverse Compton component.

The spectrum observed by MAGIC was corrected for intergalactic absorption using the recent EBL model by Dominguez et al. (2010) adopted here throughout. In the original publication of the 2006 observations the EBL model by Primack et al. (2005) was used, which underestimates the IR background according to observations (e.g. Fazio et al. 2004; Chary et al. 2004). However, as discussed in Dominguez et al. (2010), the difference between the EBL models is smaller than the systematic uncertainties of the MAGIC data analysis.

\subsection{SED February 2006}

We try to reproduce the revised data with a simple onezone leptonic emission model for FSRQs (details in Maraschi \& Tavecchio 2003) considering the synchrotron and inverse Compton (IC) emission from a population of relativistic electrons in a single emission region (spherical, with radius $R$ ) in motion with bulk Lorentz factor $\Gamma$ at an angle $\theta$ with the line of sight. The electron energy distribution is described by a smoothed broken-power law with normalization $K$ (measuring the number density of electrons at $\gamma=1$ ) extending from $\gamma_{1}$ to $\gamma_{2}$, with indices $n_{1}$ and $n_{2}$ below and above the break at a $\gamma_{b}$, respectively. The magnetic field with intensity $B$ is supposed to be homogeneous and tangled. The seed photons for the IC emission are both the synchrotron photons produced within the jet (SSC mechanism) and those outside the jet. We consider two cases, in which the IC process occurs within or outside the BLR. In the first case the high energy emission is dominated by comptonization of the UV photons of the BLR (EC/BLR). In the latter case the external radiation field is dominated by the IR thermal emission from the (putative) dusty torus (EC/IR, Ghisellini \& Tavecchio 2008; Sikora et al. 2009). In the case of the BLR emission we assume that the clouds are located at a distance $R_{\mathrm{BLR}}$ from the central black hole. The resulting emission is modeled as a black body peaking at $10^{15} \mathrm{~Hz}$ (Tavecchio \& Ghisellini 2009) with luminosity fixed to a fraction $\tau_{\mathrm{BLR}}$ of the disk luminosity (fixed to $L_{\mathrm{D}}=2 \times 10^{45} \mathrm{erg} \mathrm{s}^{-1}$, Pian et al. 1999). In the case of the torus emission, located at a distance $R_{\mathrm{IR}}$ we assume that a fraction $\tau_{\mathrm{IR}}=0.5$ of the disk luminosity is intercepted and re-radiated from dust as IR emission (again, we assume a black body spectrum, with temperature $T_{\mathrm{IR}}=10^{3} \mathrm{~K}$, see Nenkova et al. 2008).

In Fig. 8 the blue line indicates the SED obtained applying the first model (EC/BLR), while the red line corresponds to the EC/IR case. The model parameters are listed in Table 4.

The main difference between the two models (BLR and IR) is related to the different frequency of the target photons, determining the energy threshold above which the IC process is strongly suppressed due to the onset of the Klein-Nishina (hereafter $\mathrm{KN}$ ) regime. Basically, this energy can be written as (Tavecchio \& Ghisellini 2009; Ghisellini \& Tavecchio 2009):

$E_{\mathrm{KN}}=\frac{22.5}{v_{o, 15}} \frac{\delta}{\Gamma(1+z)} \mathrm{GeV}$

where $\delta$ is the relativistic Doppler factor $(\delta=\Gamma$ for $\theta=1 / \Gamma)$ and $v_{o, 15}$ is the frequency of the target photons (in units of $10^{15} \mathrm{~Hz}$ ). Therefore, if the IC process takes place within the BLR the emission in the MAGIC band is strongly affected. The KN effect is more moderate if the IC scattering takes place outside the BLR. In this case the dominant photon frequency is $v_{\mathrm{o}}=10^{13} \mathrm{~Hz}$ corresponding to $T_{\mathrm{IR}}=10^{3} \mathrm{~K}$; hence $E_{\mathrm{KN}} \simeq 300 \mathrm{GeV}$. Accordingly, somewhat harder spectra are possible in the MAGIC band. 


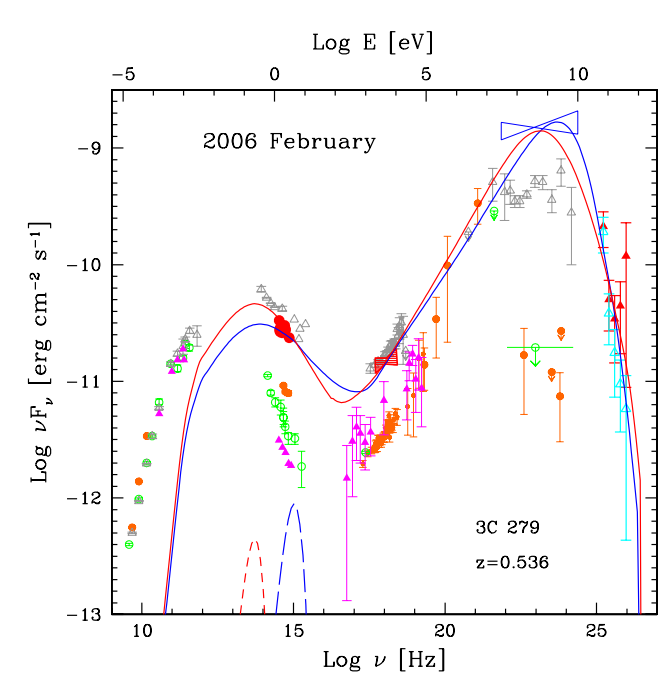

Fig. 8. The spectral energy distribution of 3C 279 on February 23, 2006. The red symbols show the optical (filled circles), RXTE (bowtie) Böttcher (2009) and MAGIC deabsorbed data (triangles) used to fit the two model curves: blue line assuming EC inside the BLR and red line EC outside the BLR. Also MAGIC observed data is shown (cyan). For comparison also historical data are shown: 1991 high state (gray: Hartman et al. 1996), 1993 low state (green: Maraschi et al. 1993) 1996 high state (orange, blue bow-tie: Ballo et al. 2002; Wehrle et al. 1998) and 2003 low state (magenta: Collmar et al. 2004). The dashed lines correspond to blackbody radiation from the IR torus (red) and BLR (blue).

The modeling of the VHE $\gamma$-ray emission from 3C 279 is particularly challenging (Albert et al. 2008a; Böttcher et al. 2009). Indeed, due to the reduced IC scattering efficiency at high energy (e.g. Tavecchio \& Ghisellini 2009) one zone emission models predict a low and rather soft emission in the VHE $\gamma$-ray band.

An additional difficulty for the BLR model is the possibility that VHE photons will be further reduced due to the internal $\gamma \gamma \rightarrow \mathrm{e}^{+} \mathrm{e}^{-}$absorption with the UV photons of the BLR (Liu et al. 2008; Sitarek \& Bednarek 2008; Tavecchio \& Mazin 2009). In contrast, when the emission occurs outside the BLR, photons with $E<1 \mathrm{TeV}$ are not substantially absorbed (above $1 \mathrm{TeV}$ they will be absorbed interacting with the IR radiation of the torus). Summarizing, these observations are very difficult to reconcile with the one-zone framework. Both the EC/BLR and the EC/IR models can reproduce the data but require a rather large flux in the LAT band. Future detections of 3C 279 simultaneously in the LAT and VHE band will be crucial to confirm or rule out this possibility.

\subsection{SED January 2007}

At the time of the second MAGIC detection, in January 2007, 3C 279 was in a brighter optical state but in a fainter X-ray state than at the discovery (February 23, 2006). Motivated by the difficulties of the one-zone model discussed above (for 2007 the required GeV flux would be even higher than for 2006), we consider two-zone model and lepto-hadronic model for this dataset.

\subsubsection{Two-zone model}

In "two zone" model, the emission from the optical up to the $\mathrm{X}$-ray and $\gamma$-ray bands derives from a different emission region than the VHE $\gamma$-ray photons. As the VHE $\gamma$-ray flare follows the optical one, we assume that the optical up to the X-ray and $\gamma$-ray emission zone is closer to central engine than the VHE $\gamma$-ray emission. Abdo et al. (2010) show that the $\gamma$-ray emission region is located on the parsec scale jet and we therefore model the spectral energy distribution assuming that the optical up to the X-ray and $\gamma$-ray bands are emitted within the BLR while the VHE $\gamma$-ray emission origins in a region outside the BLR. This also minimizes the effect of the $\mathrm{KN}$ regime and internal absorption of $\gamma$-rays. However, the modeling is valid also for emission regions further out in the jet as far as it is still located within IR torus to provide enough seed photons.

Assuming two regions we are doubling the number of free parameters, therefore this scenario is far less constrained than one-zone models. Clearly the magnetic field and particle density will be lower and the size larger for the more external region. The parameters for the two regions are reported in Table 4 and the fit to 2007 data in Fig. 9. The two-zone model can better reproduce the MAGIC data, due to the possibility to shift the peak of the EC bump to higher energies.

It is also interesting to speculate about the possible connection between the energetic flare observed in the IR band and the VHE event, following the former by almost two days. In the framework of the two-zone model, an appealing picture assumes that the jet perturbation (possibly a shell) responsible for the IR flare (assumed to be produced inside the BLR), travels and eventually reaches the region where VHE photons can be more easily produced and can escape the emission region. From the delay between the two events we can infer the distance between the location of the infrared to optical synchrotron and VHE emission zones, $d \simeq c t_{\mathrm{lag}} \Gamma^{2} \sim 2 \times 10^{18} \mathrm{~cm}$, where we assume that the perturbations travels with a Lorentz factor $\Gamma \simeq 20$, the value assumed in the radiative model. Interestingly, this distance is very well consistent with the assumed size of the VHE $\gamma$-ray emission zone.

\subsubsection{Lepto-hadronic model}

Although hadronic models in general are not favored for luminous quasars (Sikora et al. 2009), they might be viable for $3 \mathrm{C} 279$ as it does not have as hard $\mathrm{X}$-ray spectrum $(\Gamma<0.5$, for $3 \mathrm{C} 279 \Gamma=0.66$ Ballo et al. 2002) as other luminous quasars. Furthermore, models taking into account both leptonic and hadronic processes could be viable for describing the spectral energy distribution of quasars. Therefore, we also fit the data with a lepto-hadronic model. The model will be described in full detail in a forthcoming paper (Rüger et al., in prep.). It comprises a non-thermal proton and electron distribution injected into the radiation volume as a power law with lower and upper bound ( $\gamma_{\min }$ and $\gamma_{\max }$ respectively). The particles may radiate through a number of channels: The electrons may radiate through the above described mechanisms of synchrotron emission and inverse Compton scattering off synchrotron photons. The protons will also emit synchrotron radiation. Additionally they take part in photo-hadronic processes. Those are modeled by the cross-sections described in Kelner \& Aharonian (2008). In the photo-hadronic processes $\pi^{0}$ and $\pi^{ \pm}$are produced. The $\pi^{0}$ decay into $\gamma$-rays may eventually start a pair cascade in the source if $\gamma$-rays are emitted in the opticallythick regime. The $\pi^{ \pm}$decays into electrons and positrons which again emit synchrotron radiation. The time evolution is solved selfconsistently with a scheme described in Rüger et al. (2010a) for the pure leptonic case.

In this model the number of free parameters is comparable to one-zone SSC models. We introduce four extra parameters 
Table 4. Input parameters for the leptonic emission models.

\begin{tabular}{|c|c|c|c|c|c|c|c|c|c|c|c|c|c|}
\hline Model & $\gamma_{\min }$ & $\begin{array}{c}\gamma_{\mathrm{b}} \\
{\left[10^{3}\right]}\end{array}$ & $\begin{array}{l}\gamma_{\max } \\
{\left[10^{5}\right]}\end{array}$ & $n_{1}$ & $n_{2}$ & $\begin{array}{c}B \\
{[\mathrm{G}]}\end{array}$ & $\begin{array}{c}K \\
{\left[10^{4} \mathrm{~cm}^{-3}\right]}\end{array}$ & $\begin{array}{c}R \\
{\left[10^{16} \mathrm{~cm}\right]}\end{array}$ & $\delta$ & $\begin{array}{c}\theta \\
{[\mathrm{deg}]}\end{array}$ & $\tau_{\text {BLR }}$ & $\begin{array}{c}R_{\mathrm{BLR}} \\
{\left[10^{17} \mathrm{~cm}\right]}\end{array}$ & $\begin{array}{c}R_{\mathrm{IR}} \\
{\left[10^{18} \mathrm{~cm}\right]}\end{array}$ \\
\hline 2006 One-zone BLR & 1 & 2.5 & 3.5 & 2 & 3.7 & 0.15 & 2 & 5 & 20 & 2.9 & 0.015 & 4 & - \\
\hline 2006 One-zone IR & 1 & 2 & 2 & 2 & 4 & 0.19 & 1 & 4.5 & 27 & 2 & - & - & 4 \\
\hline 2007 Two-zone: Opt-X-ray zone & 1 & 0.5 & 0.03 & 2 & 4.3 & 2.2 & 5.5 & 5 & 18 & 3.1 & 0.05 & 6 & - \\
\hline 2007 Two-zone: VHE zone & 45 & 20 & 5 & 2 & 4.3 & 0.1 & 0.01 & 10 & 18 & 3.1 & - & - & 2.5 \\
\hline 2009 One-zone BLR & 1 & 0.33 & 0.2 & 2 & 3.5 & 0.8 & 23 & 3 & 20 & 2.1 & 0.1 & 6 & - \\
\hline
\end{tabular}

Notes. See text for definitions.

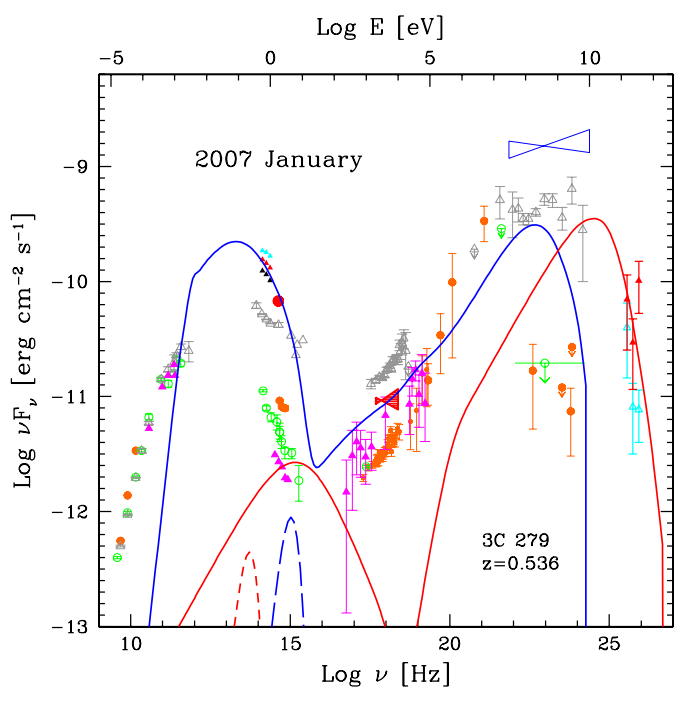

Fig. 9. The spectral energy distribution of 3C 279 on January 2007 16th (MJD 54 116). The red symbols show the REM (MJD 54 114.4, triangles), KVA (filled circle), RXTE (bow-tie) and MAGIC (deabsorbed, triangles) data used to fit the two-zone model (see text). Blue line shows the emission from the zone inside BLR and red line the emission from outside the BLR. Additionally REM data from MJD 54113.3 (Maximum of the REM lightcurve) cyan and MJD 54117.3 (black) are shown. The dashed lines correspond to blackbody radiation from the IR torus (red) and BLR (blue). The historical data as in Fig. 8.

to describe the proton population. Since we assume identical minimum Lorentz factor and spectral index for electrons and protons, we end up with a total of nine free parameters. Unlike the one-zone SSC model the variability patterns are far more complicated. In this model the lower limit of variability is still given by the beamed light crossing time, which is of the order of 4 days. Since electrons and protons may burst differently and at different times, the determination of the variability pattern is complicated. If electron and proton density are increased simultaneously, the electrons produce the well-known soft lag pattern in the electron synchrotron radiation and the inverse Compton radiation. In the hadronic component this is also true for the synchrotron radiation. For the $\pi$ production intermediate secondary energies peak earlier.

To model the data (fit shown in Fig. 10) we used electron and proton spectra with a $\gamma_{\min }=150$ and a spectral index $s=2.2$. For the protons we find $\gamma_{\max }=10^{9}$, while the electrons have a much lower $\gamma_{\max }=5 \times 10^{4}$. Spectral breaks are calculated selfconsistently from the loss processes within the radiation zone. The magnetic field is assumed to be $0.025 \mathrm{G}$ with a source radius of $5.3 \times 10^{17} \mathrm{~cm}$. The doppler factor is 42 . The energy density of the electrons is one order of magnitude higher than the magnetic field energy density, while the proton energy density is

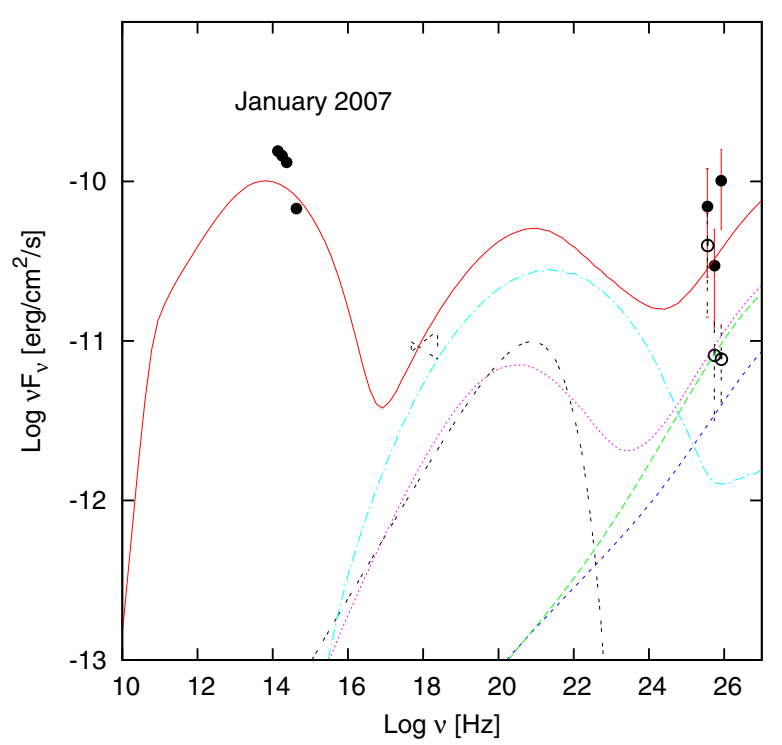

Fig. 10. The observed spectral energy distribution of January 2007 modeled with the lepto-hadronic model. The overall fit is shown with red line with the following components at high energies: the synchrotron radiation of pair creation electrons and positrons cascaded down from the optically thick regime (magenta dotted line), synchrotron radiation from positrons from the pion decay (green dashed line), synchrotron radiation from electrons from the pion decay (blue dashed line), inverse Compton scattering (cyan dot dashed line) and proton synchrotron emission (black double dashed line). The VHE $\gamma$-ray emission is mostly sum of the three first components while in X-rays the main contribution comes from the inverse Compton scattering (like in the purely leptonic models). The low energy bump is produced by the electron synchrotron radiation.

already six orders of magnitude higher. Even though this is not in equilibrium this might still be confined, as the proton gyroradius at highest energies is of the order of the blob radius.

The parameters suggest that the radiation zone may be close to the end of magnetic confinement zone, $\sim 1000$ Schwarzschild radii from the supermassive black hole. However, the model itself is not limited to that region, but may be used on every point along the jet axis. Although internal pair absorption is included, external photons producing pairs are not included in the model and therefore if the emission region was inside the BLR the observed flux would be reduced.

\subsection{SED January 2009}

For the 2009 observations we present a SED built using multiwavelength data nearly simultaneous to the MAGIC data taken in the period 21-31 January, which yielded in an upper limits in the VHE $\gamma$-ray band. $\gamma$-ray data were derived from Fermi LAT and averaged over the same period (Fermi data were not 


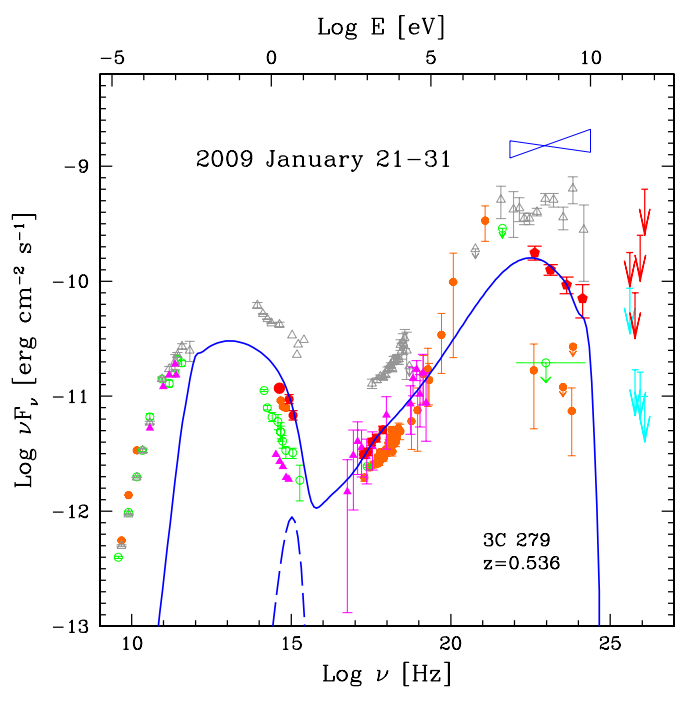

Fig. 11. The spectral energy distribution of 3C 279 on January 2009: MAGIC (taken in the period 21-31 January, red arrows: deabsorbed, cyan arrows: observed), LAT (averaged over the same period, red pentagons), XRT and UVOT (February 1, 2009 red squares) and KVA (January 25, 2009 red filled circle). The SED is modeled assuming the emission region inside the BLR. The historical data as in Fig. 8. The dashed line correspond to blackbody radiation from the IR torus (red).

available at the previous epochs) and XRT and UVOT data of February 1, 2009 were used. The source was in a rather low state at all available bands. The SED can be modeled quite satisfactorily with a standard one zone model assuming the emission region inside the BLR (Fig. 11) with typical parameters (reported in Table 4).

\section{Summary and conclusions}

In this paper we report the MAGIC observations of 3C 279 in January 2007 and from December 2008 to April 2009. The January 2007 observations yielded a detection on the night of January 16, when the source was in a high optical state. Like in February 2006 when the source was discovered, in January 2007 the source was detected on one night only. However, in January 2007 MAGIC observed the source also in the previous and the following night and these observations resulted in non-detection, which confirms the day-scale variability of the source.

In February 2006 and January 2007 the source was in a high optical-IR state and was also showing a rather high state in X-rays. However, no major increase in the optical or X-ray flux was seen simultaneously to the VHE $\gamma$-ray flare. Unlike in February 2006 and January 2007, in January 2009 the source was observed by MAGIC during a low optical-X-ray state. The existence of LAT data for this epoch shows that the source was relatively faint also at $\mathrm{GeV}$ energies. Consistently, the MAGIC observations resulted in upper limits.

In the spring of 2009 Fermi detected a fast $\gamma$-ray flare from 3C 279 which was coincident with a rotation of the optical polarization angle of 180 degrees that had duration of $\sim 20$ days. In early 2007 such rotation of the polarization angle also took place (Larionov et al. 2008), but with rotation of $\sim 360$ degrees, the duration of rotation $\sim 2$ times longer and the direction of the rotation opposite to what was seen in 2009. The MAGIC detection in January 2007 took place in the beginning of the rotation of optical polarization angle, thus confirming that rotation of optical polarization angle in 3C 279 are recurrently accompanied with $\gamma$-ray flares. This seems to be common in FSRQs, the same behavior has been observed in PKS 1510-089 in 2009 (Marscher et al. 2010).

However, the patterns in 3C 279 in January 2007 and February 2009 are clearly different, which might suggest that different mechanisms for producing the rotation of the polarization angle could be in action (e.g. for 2009 the rotation could be produced by turbulence, see e.g. D'Arcangelo et al. 2007). Therefore it is not possible to draw definite conclusions on the connection between the rotation of polarization angle with $\gamma$-ray flares either.

We find that the spectral energy distribution of 3C 279 inJanuary 2007 cannot be explained by the standard one-zone $\mathrm{SSC}+\mathrm{EC}$ model, where the emission region is located inside the BLR which provides the seed photons for inverse Compton scattering. Instead we explore a two-zone model, where the VHE $\gamma$-ray emitting region is located just outside the BLR, while the standard optical-to-X-ray and $\gamma$-ray emitting region is still inside the BLR region and a lepto-hadronic model, which both fit the data reasonably well. Also other possible scenarios for the emission exists. Sitarek \& Bednarek (2010) suggested a leptonic cascade model. Several authors have also suggested that blazar emission zone would be located at parsec scale distances from the black hole: there $\gamma$-rays could be produced via up-scattering of infrared photons from the hot dust (Blazejowski et al. 2000; Sikora et al. 2009). It has also been suggested that the relativistic jet could drag part of the BLR clouds to large distances from the central engine (at least in radio galaxies: Arshakian et al. 2010; Leon-Tavares et al. 2010a) and in principle these photons could serve as external seed photons for inverse Compton scattering (Leon-Tavares et al. 2011). The multiwavelength behavior of the source in January 2007 can also be interpreted in favor of emission zone beyond VLBI core and in such case multi emission shell model suggested by Marscher et al. (2010) could be viable.

After the first detection of 3C 279 by MAGIC, two other flat spectrum radio quasars have been detected in the VHE $\gamma$-ray band, PKS 1510-089 ( $z=0.36$, Wagner et al. 2010) and PKS 1222+21 $(z=0.432$, Mariotti et al. 2010; Aleksić et al. 2011). In all the cases the emission of the VHE $\gamma$-ray radiation poses problems to the standard models, as discussed in this paper and in Aleksić et al. (2011). Future multiwavelength observations, including higher sensitivity observations with MAGIC II, will be needed to improve our understanding of the high-energy emission from FSRQs.

Acknowledgements. We would like to thank the Instituto de Astrofísica de Canarias for the excellent working conditions at the Observatorio del Roque de los Muchachos in La Palma. The support of the German BMBF and MPG, the Italian INFN, the Swiss National Fund SNF, and the Spanish MICINN is gratefully acknowledged. This work was also supported by the Marie Curie program, by the CPAN CSD2007-00042 and MultiDark CSD2009-00064 projects of the Spanish Consolider-Ingenio 2010 programme, by grant DO02-353 of the Bulgarian NSF, by grant 127740 of the Academy of Finland, by the YIP of the Helmholtz Gemeinschaft, by the DFG Cluster of Excellence "Origin and Structure of the Universe", and by the Polish MNiSzW Grant N N203 390834. Authors wish to thank Alan Marscher for useful discussions and the referee Elena Pian for useful comments on the earlier version of the paper.

\section{References}

Abdo, A., Ackermann, A. M., Ajello, M., et al. (the Fermi LAT Collaboration) 2009, ApJ, 700, 597

Abdo, A., Ackermann, A. M., Ajello, M., et al. (the Fermi LAT Collaboration and Members of the 3C 279 Multi-Band Campaign) 2010, Nature, 463, 919 Agudo, I., Jorstad, S., Marscher, A., et al. 2011, ApJ, 726, L13

Aharonian, F., Akhperjanian, A. G., Anton, G., et al. (the HESS Collaboration) 2009, A\&A, 502, 749 
Albert, J., Aliu, E., Anderhub, H., et al. (the MAGIC Collaboration) 2007, ApJ, 669,862

Albert, J., Aliu, E., Anderhub, H., et al. (the MAGIC Collaboration) 2008a, Science, 320, 1752

Albert, J., Aliu, E., Anderhub, H., et al. (the MAGIC Collaboration) 2008b, ApJ, 674,1037

Albert, J., Aliu, E., Anderhub, H., et al. (the MAGIC Collaboration) 2008c, Nucl. Instr. Meth. A, 594, 407

Albert, J., Aliu, E., Anderhub, H., et al. (the MAGIC Collaboration) 2008d, Nucl. Instr. Meth. A, 588, 424

Aleksić, J., Anderhub, H., Antonelli, L. A., et al. (the MAGIC Collaboration) 2010, A\&A, 519, 32

Aleksić, J., Antonelli, L. A., Antoranz, P., et al. (the MAGIC Collaboration) 2011, ApJL, 730, 8

Aliu, E., Anderhub, H., Antonelli, L. A., et al. (the MAGIC Collaboration) 2009, Astropart. Phys., 30, 293

Arshakian, T. G., Leon-Tavares, J., Lobanov, A. P., et al. 2010, MNRAS, 401, 1231

Ballo, L., Maraschi, L., Tavecchio, F., et al. 2002, ApJ, 567, 50

Bessell, M. S. 1979, PASP, 91, 589

Blazejowski, M., Sikora, M., Moderski, R., \& Madejski, G. M. 2000, ApJ, 545, 107

Böttcher, M., Reimer, A., \& Marscher, A. 2009, ApJ, 703, 1168

Bretz, T., \& Dorner, D. (MAGIC Collab.) 2008, AIP Conf. Proc., 1085, 664

Bretz, T., \& Wagner, R. 2003, in Proceedings of the 28th International Cosmic Ray Conference, Tsukuba, Japan, 5, 2947

Britgzer, D., Carmona, E., Majumdar, P., et al. 2009, Proc. 31st ICRC, Lodz, Poland, July 2009 [arXiv: 0907.0973]

Chatterjee R., Jorstad, S. G., \& Marscher, A. 2008, ApJ, 689, 79

Chary, R., Casetarno, S., \& Dickinson, M. E. 2004, ApJS, 154, 80

Chincarini, G., Zerbi, F. M., Antonelli, L. A., et al. 2003, The Messenger, 113, 40

Ciprini, S., \& Chaty, S. (Fermi Collaboration) 2008, ATel, 1864

Collmar, W., et al. 2004, Proc. 5th INTEGRAL Workshop on the INTEGRAL

Universe (ESA SP-552), 16-20 February, Munich, Germany. ed. V. Schönfelder, G. Licht, \& C. Winkler, 555

Collmar, W., Böttcher, M., Krichbaum, T., et al. 2010, A\&A, 522, 66

Covino, S., Zerbi, F. M., Chincarini, G., et al. 2004a, AN, 325, 543

Covino, S., Stefanon, M., Sciuto, G., et al. 2004b, SPIE, 5492, 1613

Daum, A., Hermann, G., Hess, M., et al. (The HEGRA Collaboration) 1997, $\mathrm{APh}, 8,1$

D'Arcangelo, F., Marscher, A. P., Jorstad, S., et al. 2007, ApJ, 659, 107

Degl'Innocenti, E. L., Bagnulo, S., \& Fossati, L. 2007, in The Future of Photometric, Spectrophotometric, and Polarimetric Standardization, ed. C. Sterken, ASP, 364, 495

Dermer, C. D., \& Schlickeiser, R. 1993, ApJ, 416, 458

Devillard, N. 1997, The Messenger, 87

Domínguez, A., Primack, J. R., Rosario, D. J., et al. 2010, MNRAS, 410, 2556

Fazio G. G., Ashby, M. L. N., Barmby, P., et al. 2004, ApJS, 154, 39

Fomin, V. P., Stepanian, A. A., Lamb, R. C., et al. 1994, Astropart. Phys., 2, 137

Gehrels, N., Chincarini, G., Giommi, P., et al. 2004, ApJ, 611, 1005

Ghisellini, G., \& Tavecchio, F. 2008, MNRAS, 387, 1669

Ghisellini, G., \& Tavecchio, F. 2009, MNRAS, 397, 985

Hartman, R. C., Bertsch, D. L., Fichtel, C. E., et al. 1992, ApJ, 385, 1

Hartman, R. C., Webb, J. R., Marscher, A. P., et al. 1996, ApJ, 461, 698

Hartman, R. C., Villata, M., Balonek, T. J., et al. 2001, ApJ, 558, 583

Hillas, A. M. 1985, in Proc. 19th International Cosmic Ray Conference, La Jolla, 3,445

Hewitt, A., \& Burbidge, G. 1993, ApJS, 87, 451

Jorstad, S. G., Marscher, A. G., Mattox J. R., et al. 2001, ApJ, 556, 738

Jorstad, S. G., Marscher, A. P., Lister, M. L., et al. 2004, AJ, 127, 3115

Jorstad, S., Marscher, A., D’Arcangelo, F., \& Harrison, B. 2010, 2009 Fermi Symposium and eConf Proceedings [arXiv: 0912.5230]

Kalberla, P. M. W., Burton, W. B., Hartmann, D., et al. 2005, A\&A, 440, 775

Kelner, S. R., \& Aharonian, F. A. 2008, Phys. Rev. D., 78, 4013

Leon-Tavares, J., Lobanov, A. P., Chavushyan, V. H., et al. 2010, ApJ, 715, 355

Leon-Tavares, J., Valtaoja, E., Tornikoski, M., Lähteenmäki, A., \& Nieppola, E. 2011, A\&A, Submitted [arXiv: 1102 .1290v1]

Lessard, R. W., Buckley, J. H., Connaughton, V., et al. 2001, Astropart. Phys., 15,1

Li,T.-P., \& Ma, Y.-Q. 1983, ApJ, 272, 317

Lindfors, E., Türler, M., Valtaoja, E., et al. 2006, A\&A, 456, 895

Liu, H. T., Bai, J. M., \& Ma, L. 2008, ApJ, 688, 148

Mannheim, K., \& Biermann, P. L. 1992, A\&A, 253, L21

Maraschi, L., \& Tavecchio, F. 2003, ApJ, 593

Maraschi, L., Ghisellini, G., \& Celotti, A. 1992, ApJ, 397, L5

Maraschi, L., Grandi, P., Urry, C. M., et al. 1994, ApJ, 435, 91

667
Marscher, A. P., \& Jorstad, S. G. 2010, in Proc. Fermi meets Jansky - AGN in Radio and Gamma-Rays, ed. T. Savolainen, E. Ros, R. W. Porcas, \& J. A. Zensus

Marscher, A. P., Jorstad, S. G., Larionov, V., et al. 2010, ApJ, 710, L126

Mariotti, M. (for the MAGIC Collaboration) 2010, ATel, 2684

Mattox, J. R., Bertsch, D. L., Chiang, J., et al. 1996, ApJ, 461, 396

Mücke, A., Protheroe, R. J., Engel, R., et al. 2003, APh, 18, 593

Nenkova, M., Sirocky, M., Ivezic, Z., \& Elitzur, M. 2008, ApJ, 685, 147

Pei, Y. C. 1992, ApJ, 395, 130

Pian, E., Urry, C. M., Maraschi, L., et al. 1999, ApJ, 521, 112

Poole, T. S., Poole, T. S., Breeveld, A. A., Page, M. J., et al. 2008, MNRAS, 383, 627

Primack, J. R., Bullock, J. S., \& Somerville, R. S. 2005, in High Energy GammaRay Astronomy, ed. F. Aharonian, H. Voelk, D. Horns (AIP, Heidelberg), AIP Conf. Ser., 745, 23

Raiteri, C. M., Villata, M., Lanteri, L., Cavallone, M., \& Sobrito, G. 1998, A\&AS, 130, 495

Riegel, B., Bretz, T., Dorner, D., et al. (MAGIC Collab.) 2005, in Proc. 29th International Cosmic Ray Conference, Pune, India, 5, 215

Rüger, M., Spanier, F., \& Mannheim, K. 2010, MNRAS, 401, 973

Schlegel, D. J., Finkbeiner, D. P., \& Davis, M. 1998, ApJ, 500, 525

Sikora, M., Begelman, M. C., \& Rees, M. J. 1994, ApJ, 421, 153

Sikora, M., Blazejowski, M., Madejski, G., et al. 2001, ESASP, 459, 259S

Sikora, M., Moderski, R., \& Madejski, G. M. 2008, ApJ, 675, 71

Sikora, M., Stawarz, L., Moderski, R., Nalewajko, K., \& Madejski, G. 2009, ApJ, 704, 38

Sitarek, J., \& Bednarek, W. 2008, MNRAS, 391, 624

Sitarek, J., \& Bednarek, W. 2010, MNRAS, 409, 662

Tavecchio, F., \& Ghisellini, G. 2008, MNRAS, 386, 28

Tavecchio, F., \& Ghisellini, G. 2009, MNRAS, 394, 131

Tavecchio, F., \& Mazin, D. 2009, MNRAS, 392, 40

Turnsheak, D. A., Bohlin, R. C. Williamson, R. L., et al. 1990, AJ, 99, 1243

Wagner, S. J. (the HESS collaboration) 2010, HEAD Meeting, Hawaii (BAAS, 42, 2, 07.05)

Wehrle, A., Pian, E., Urry, C. M., et al. 1998, ApJ, 497, 178

Zerbi, F. M., Chincarini, G., Ghisellini, G., et al. 2001, AN, 322, 275

1 IFAE, Edifici Cn., Campus UAB, 08193 Bellaterra, Spain

2 INAF National Institute for Astrophysics, 00136 Rome, Italy e-mail: fabrizio.tavecchio@brera.inaf.it

3 Università di Siena, and INFN Pisa, 53100 Siena, Italy

4 Technische Universität Dortmund, 44221 Dortmund, Germany

5 Universidad Complutense, 28040 Madrid, Spain

6 Università di Padova and INFN, 35131 Padova, Italy

7 Inst. de Astrofísica de Canarias, 38200 La Laguna, Tenerife, Spain e-mail: berger@astro.uni-wuerzburg.de

8 Depto. de Astrofísica, Universidad de La Laguna, 38206 La Laguna, Spain

9 University of Łódź, 90236 Łódź, Poland

10 Tuorla Observatory, University of Turku, 21500 Piikkiö, Finland e-mail: elilin@utu.fi

11 Deutsches Elektronen-Synchrotron (DESY), 15738 Zeuthen, Germany

12 ETH Zurich, 8093 Switzerland

13 Max-Planck-Institut für Physik, 80805 München, Germany

14 Universität Würzburg, 97074 Würzburg, Germany

15 Universitat de Barcelona (ICC/IEEC), 08028 Barcelona, Spain

16 Università di Udine, and INFN Trieste, 33100 Udine, Italy

17 Institut de Ciències de 1'Espai (IEEC-CSIC), 08193 Bellaterra, Spain

18 Inst. de Astrofísica de Andalucía (CSIC), 18080 Granada, Spain

19 Croatian MAGIC Consortium, Institute R. Boskovic, University of Rijeka and University of Split, 10000 Zagreb, Croatia

20 Universitat Autònoma de Barcelona, 08193 Bellaterra, Spain

21 Inst. for Nucl. Research and Nucl. Energy, 1784 Sofia, Bulgaria

22 INAF/Osservatorio Astronomico and INFN, 34143 Trieste, Italy

23 Università dell'Insubria, Como, 22100 Como, Italy

24 Università di Pisa, and INFN Pisa, 56126 Pisa, Italy

25 ICREA, 08010 Barcelona, Spain

26 University of California, Los Angeles, USA 